\title{
Descriptions of species of Stegelleta Thorne, 1938 (Nematoda, Rhabditida, Cephalobidae) from California, New Zealand and Senegal, and a revision of the genus
}

\author{
Sven BOSTRÖM ${ }^{1} \&$ Oleksandr HOLOVACHOV ${ }^{2}$ \\ ${ }^{1,2}$ Department of Zoology, Swedish Museum of Natural History, Box 50007, \\ SE-104 05 Stockholm, Sweden \\ ${ }^{1}$ Email: sven.bostrom@nrm.se (corresponding author) \\ ${ }^{2}$ Email: oleksandr.holovachov@nrm.se \\ ${ }^{1}$ urn:lsid:zoobank.org:author:528300CC-D0F0-4097-9631-6C5F75922799 \\ ${ }^{2}$ urn:Isid:zoobank.org:author:89D30ED8-CFD2-42EF-B962-30A13F97D203
}

\begin{abstract}
Populations of Stegelleta are described from California, New Zealand and Senegal. An amphimictic population from California is identified as belonging to $S$. incisa and compared with type specimens from Utah and an amphimictic population from Italy. One population from New Zealand is close to $S$. incisa but considered to represent a new species, Stegelleta laterocornuta sp. nov. It is particularly characterised by a 379-512 $\mu \mathrm{m}$ long body in females and 365-476 $\mu \mathrm{m}$ in males; cuticle divided into 16 rows of blocks at midbody (excluding lateral field); lateral field with four incisures; three pairs of asymmetrical lips, U-shaped primary axils without guarding processes, each lip asymmetrically rectangular with a smooth margin, only lateral lips have slender acute tines; three labial probolae, bifurcated at half of their length; vulva without flap; spermatheca $17-31 \mu \mathrm{m}$ long; postuterine sac 7-24 $\mu \mathrm{m}$ long; spicules $21.5-23.5 \mu \mathrm{m}$ long. Other specimens from New Zealand are identified as belonging to $S$. tuarua. A parthenogenetic population from Senegal is identified as belonging to $S$. ophioglossa and compared with type specimens from Mongolia and records of several other populations of $S$. ophioglossa. The generic diagnosis is emended and a key to the species of Stegelleta is provided.
\end{abstract}

Keywords. Morphology, new species, SEM, Stegelleta, taxonomy.

Boström S. \& Holovachov O. 2014. Descriptions of species of Stegelleta Thorne, 1938 (Nematoda, Rhabditida, Cephalobidae) from California, New Zealand and Senegal, and a revision of the genus. European Journal of Taxonomy 87: 1-19. http://dx.doi.org/10.5852/ejt.2014.87

\section{Introduction}

The genus Stegella was erected by Thorne (1937) with Stegella incisa Thorne, 1937 as type species, for cephalobids having: cuticle divided into plate-like segments over entire body; labial probolae furcate, prongs simple or with few branches; cephalic probolae variable, rounded axil borders or flap-like with membranes; and wing area unusually broad with a medial line and crenate borders. 
Due to a homonomy with a hydroid, Stegella Stechow, 1919, it was later renamed and became Stegelleta Thorne, 1938. Stegelleta currently includes six valid species of which the most recent one, S. arenaria Boström \& Holovachov, 2012, was described from Kelso Dunes, Mojave National Preserve, California. Specimens of $S$. incisa were also found in the samples from Kelso Dunes. This population is here described from studies by light (LM) and scanning electron microscope (SEM) and compared with type specimens from Utah and an amphimictic population from Italy described under the name S. ophioglossa Andrássy, 1967 (Thorne 1937; Orselli \& Vinciguerra 2002). Another population from New Zealand, described here from studies by light and scanning electron microscope, is close to $S$. incisa but considered to represent a new species. Some specimens of $S$. tuarua Yeates, 1967 from New Zealand are also described and compared with the original description (Yeates 1967). A recent parthenogenetic population of S. ophioglossa from Senegal is described and compared with the original description of S. ophioglossa from Mongolia and records of populations from Uzbekistan, Senegal, Iran and Spain (Andrássy 1967; Mavljanov 1978; De Ley et al. 1990; Shokoohi et al. 2008; Abolafia et al. 2011). The generic diagnosis is emended and a key to the species of Stegelleta is provided.

\section{Material and methods}

During a tour in the Kelso Dunes area, Mojave National Preserve, southern California, the junior author collected several samples of sand underneath different species of desert plants. Nematodes were recovered from sand samples using a modified Baermann funnel extraction. For LM, specimens were relaxed by gentle heat, fixed in cold $4 \%$ formaldehyde solution, transferred to pure glycerine by a slow evaporation method and mounted on permanent slides in glycerine with paraffin wax as support for the coverslip. After measuring and observations, some specimens were removed from slides and rehydrated by first gradually adding drops of S1 (1\% glycerine, 20\% ethanol and 79\% distilled water) to glycerine in an embryo-dish until the volume tripled and then gradually adding distilled water until the volume tripled again. The specimens were then washed in distilled water before resuspension in formaldehyde. For SEM, specimens were post-fixed in $1 \%$ osmium tetroxide $\left(\mathrm{OsO}_{4}\right)$ and transferred to pure acetone through an acetone/distilled water series. Specimens were critical point dried in liquid $\mathrm{CO}_{2}$, mounted on stubs, gold-plated under vacuum to a thickness of $200 \AA$ in an Agar High Resolution Sputter Coater Model 20, and examined in a Hitachi S-4300 SEM at an accelerating voltage of $5 \mathrm{kV}$.

Specimens from New Zealand were collected by Christine (Gamble) Powell in the Abel Tasman National Park and subsequently cultured as the strain Stegelleta sp. JB-75, which was sequenced and included in a phylogenetic study on Cephalobina (Nadler et al. 2006). Additional material from the Castlecliff beach in New Zealand was supplied by Dieter Sturhan and Gregor Yeates. This is one of the localities where the original populations of S. iketaia Yeates, 1967 were collected. Specimens from Senegal were collected in the Kaolack region, Thyssé-Kaymor by Cécile Villenave. For LM, the specimens from New Zealand and Senegal were processed using different modifications of a slow evaporation method. Specimens from the strain Stegelleta sp. JB-75 were processed to SEM by Dan Bumbarger and photographs were put at our disposal.

Morphometric characters applied herein and their abbreviations are as defined for Cephalobidae in De Ley et al. (1999); terminology of the labial and cephalic region and stoma terminology follows Holovachov et al. (2009). Type and other specimens are deposited in the invertebrate collections of the Department of Zoology, Swedish Museum of Natural History, Stockholm, Sweden (SMNH). 


\section{Results}

Class Chromadorea Inglis, 1983

Order Rhabditida Chitwood, 1933

Family Cephalobidae Filipjev, 1934

Genus Stegelleta Thorne, 1938

Stegella Thorne, 1937: 4 [junior homonym of Stegella Stechow, 1919].

Stegelleta Thorne, 1938: 64-65.

\section{Type species}

Stegella incisa Thorne, 1937.

Diagnosis (emended after Holovachov et al. 2009)

Cuticle annulated, without distinctly annulated internal layer; annuli with longitudinal incisures (tessellated). Lateral field with two or four wings (appearing as three, four or five incisures); ending at tail terminus in females and in males. Lip region weakly offset, consisting of six globular lips arranged in three pairs: one dorsal and two subventral. Pairs of lips separated by primary axils; guarding processes absent; secondary axils undeveloped. Cephalic probolae absent or in the shape of one acute tine extending along the primary axil occurring only on lateral lips or on all six lips. Labial probolae elongate-conoid with broad basis, bifurcated about halfway forming two slender prongs, in some species further bifurcated apically. Six labial and four cephalic papilliform sensilla arranged in a cephaloboid manner. Amphidial aperture slit-shaped, located on lateral lips. Stoma divided into cheilo-, gymno- and stegostom: cheilostom barrel-shaped with strongly sclerotized bacilliform cheilorhabdia; gymnostom weakly developed, as wide as stegostom, with weak gymnorhabdia; stegostom consists of a funnel-shaped prostegostom and variably shaped mesostego-, metastego- and telostegostom parts. Metastegostom denticle present. Pharynx cephaloboid: pharyngeal procorpus and metacorpus cylindrical, lining of procorpus and metacorpus are of same thickness; isthmus narrower than metacorpus; basal pharyngeal bulb oval, with strongly developed valves. Nerve ring encircling posterior part of metacorpus or anterior part of isthmus. Excretory pore opens at level of nerve ring, at posterior part of metacorpus or anterior part of isthmus. Deirids present. Female reproductive system cephaloboid; posterior part of ovary straight; spermatheca present; postvulval uterine sac present; vagina straight; vulva flat, vulval flap absent or present. Male reproductive system cephaloboid; spicules cephaloboid with manubrium and corpus of approximately equal width; gubernaculum plate-like or wedge-shaped; cornua crurum present. Male genital papillae: at least one ventrosublateral pair located anterior to cloaca; one ventrosublateral pair located at the level of cloacal opening; two pairs located at middle of tail length; and three pairs (lateral, subventral and subdorsal) near tail terminus; there is a midventral papilla on anterior cloacal lip. Rectum short (1-2 times longer than anal body diameter). Phasmids located at about one-third to half of tail length in both sexes. Female tail conoid or subcylindrical, straight, tail terminus bluntly rounded or truncate; male tail conoid, arcuate ventrad, tail terminus bluntly rounded.

\section{Valid species}

Stegelleta arenaria Boström \& Holovachov, 2012

Stegelleta georgica Bagaturija, 1973

Stegelleta iketaia Yeates, 1967

Stegelleta incisa (Thorne, 1937)

Stegelleta ophioglossa Andrássy, 1967

= Stegelleta cylindrica Mavljanov, 1978: 1889, figs 1-6

Stegelleta tuarua Yeates, 1967 
Stegelleta incisa (Thorne, 1937)

Figs 1A-E, 2, Table 1

Stegella incisa Thorne, 1937: 14-15, fig. 4G-I.

Stegelleta incisa - Thorne 1938: 65.

Stegelleta ophioglossa - Orselli \& Vinciguerra 2002: 216-219, figs 5, 6A-C.

\section{Diagnosis}

Stegelleta incisa is characterised by a $465-580 \mu \mathrm{m}$ long body in females and $428-600 \mu \mathrm{m}$ in males; cuticle divided by regular longitudinal striations into 16 rows of blocks at midbody (excluding lateral field); lateral field with four incisures extending almost to tail terminus in females and to midtail papillae in males, two outer incisures extend to tail terminus in males; three pairs of asymmetrical lips, pairs of lips separated by U-shaped primary axils without guarding processes, secondary axils demarcated by a shallow incisure, each lip asymmetrically rectangular with a smooth margin and without tines; three labial probolae, bifurcated at half of their length, prongs bent toward one another apically, "snaketongue"-shaped; pharyngeal corpus 2.1-2.9 times isthmus length; nerve ring and excretory pore at level of metacorpus to metacorpus-isthmus junction, deirids at level of metacorpus-isthmus junction to isthmus; vulva with anterior flap; spermatheca $24-43 \mu \mathrm{m}$ long; postuterine sac 22-36 $\mu \mathrm{m}$ long; spicules $22-36 \mu \mathrm{m}$ long.

\section{Material examined}

USA: 13 우, 9 ๙ึ, SMNH 135940-135942, 28 Mar. 2010, California, Mojave National Preserve, Kelso Dunes, soil around roots of desert plants $\left(34^{\circ} 53.698^{\prime} \mathrm{N}, 115^{\circ} 42.155^{\prime} \mathrm{W}\right.$ and $34^{\circ} 53.754^{\prime} \mathrm{N}, 115^{\circ}$ $42.248^{\prime}$ W), legit O. Holovachov \& P. De Ley.

\section{Description}

\section{Adult}

Body slightly arcuate in different ways when killed by heat, males often strongly arcuate ventrad in tail end. Cuticle coarsely annulated, annuli 2.4-3.4 $\mu \mathrm{m}$ wide at midbody and 2.2-3.0 $\mu \mathrm{m}$ wide in pharyngeal region. Cuticle tessellated: longitudinal striae giving it a tiled appearance, each block often with a central indentation as seen under SEM. Anteriorly, 9-10 first annuli, the longitudinal striation is irregular and blocks rounded, followed by a more regular striation creating rectangular blocks, 16 rows of blocks at midbody (excluding lateral field). Lateral field consisting of two wings separated by a broad groove, appearing as four incisures under LM, occupying about $20-25 \%$ of body diameter, extending almost to tail terminus in females and to midtail papillae in males, two outer incisures extend to tail terminus in males. Lip region slightly offset, carrying $6+4$ papillae and two round amphids. Three pairs of asymmetrical lips, one dorsal and two ventrolateral. Pairs of lips separated by U-shaped primary axils without guarding processes. Each pair with a shallow incisure demarcating a secondary axil. Each lip asymmetrically rectangular with a smooth margin, without any tines. Three labial probolae, 8.0-9.5 $\mu \mathrm{m}$ high, bifurcated at half of their length, prongs bent toward one another apically ("snake-tongue"-shaped). Stoma somewhat longer than lip region diameter. Stomatal parts not clearly discernible. Cheilorhabdia bacilliform in lateromedian view; metastegostom with a dorsal denticle. Pharynx cephaloboid. Pharyngeal corpus cylindrical; isthmus narrow, often folded, and not clearly demarcated from corpus; bulb oval, with valves. Nerve ring at level of metacorpus to metacorpus-isthmus junction. Excretory pore and deirids at level of metacorpus-isthmus junction to isthmus.

\section{Female}

Reproductive system monodelphic, prodelphic, in dextral position in relation to intestine. Ovary reflexed posteriorly at oviduct, ovary straight posterior to vulva. Spermatheca well developed. Postvulval uterine 


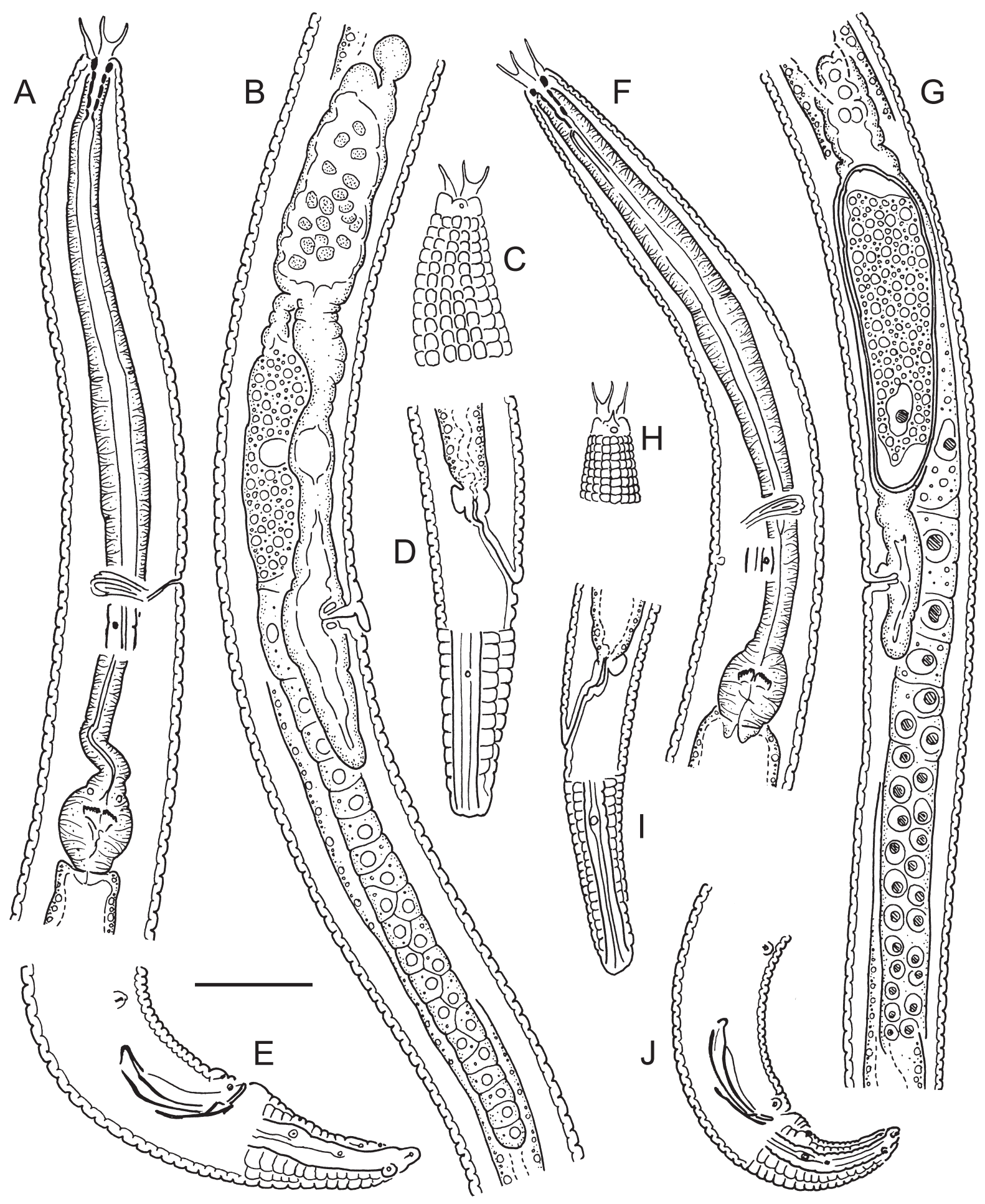

Fig. 1. A-E. Stegelleta incisa (Thorne, 1937). A. Pharyngeal region. B. Female gonad. C. Anterior end, surface view. D. Female tail. E. Male tail. F-J. Stegelleta laterocornuta sp. nov. F. Pharyngeal region. G. Female gonad. H. Anterior end, surface view. I. Female tail. J. Male tail. Scale bar $=20 \mu \mathrm{m}$. 
sac one to one-and-a-half times vulval body diameter long. Vagina about one-third of vulval body diameter. Vulval lips not or slightly protruding, anterior lip with cuticular flap directed posteriad and partly covering vulval opening. Vulva covered by genital plug in some specimens. Tail conoid with 13-15 ventral annuli, terminus truncate. One specimen has a tail with only 10-11 annuli and broadly rounded terminus. Phasmids located at about one-third to half of tail length.

\section{Male}

Reproductive system monorchic, dextral in position; testis reflexed ventrad anteriorly. Spicules paired and symmetrical, curved ventrad; with oval manubrium and subcylindrical, gradually narrowing shaft. Gubernaculum plate-like, cornua crurum present. Tail strongly arcuate ventrad, conoid with rounded terminus. The two outer lateral lines extend posterior to the phasmid, transforming into a cuticular ridge that reaches tail terminus. Genital papillae arranged as follows: two pairs subventral precloacal (at 24-33 $\mu \mathrm{m}$ and at 42-58 $\mu \mathrm{m}$ anterior to cloaca), one pair subventral adcloacal, a single midventral papilla

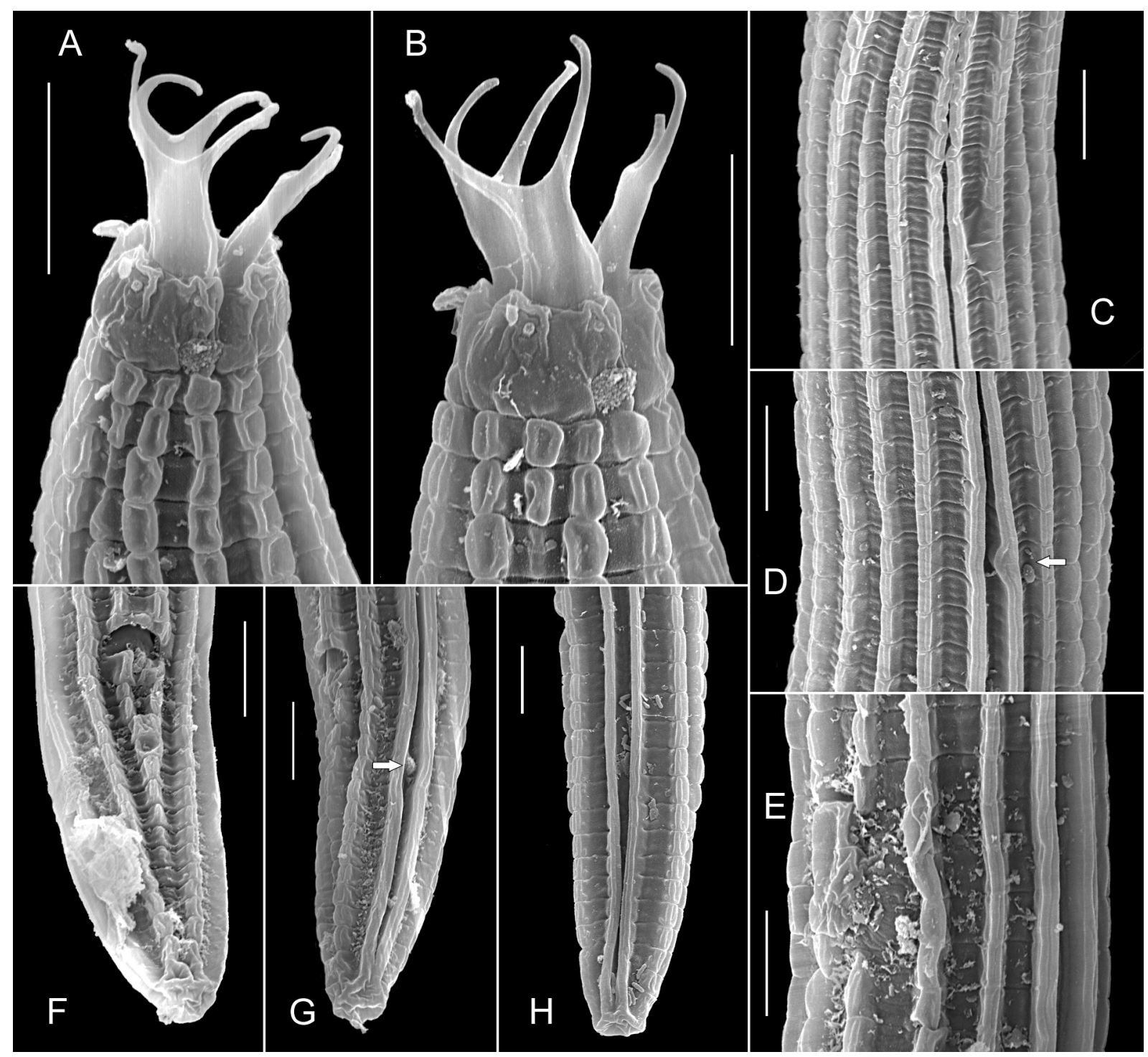

Fig. 2. Stegelleta incisa (Thorne, 1937), SEM micrographs. A-B. Anterior end, left lateral view. C. Anterior part of lateral field. D. Deirid (arrow). E. Vulval region. F. Female tail, subventral view. G. Female tail, left sublateral view (arrow points at phasmid). H. Female tail, lateral view. Scale bars $=5 \mu \mathrm{m}$. 
Table 1. Measurements (in $\mu \mathrm{m}$ ) of Stegelleta incisa (Thorne, 1937) from Kelso Dunes, California compared with the type population of S. incisa from Utah and a bisexual population described by Orselli \& Vinciguerra (2002) from Italy under the name S. ophioglossa Andrássy, 1967 (presented as mean \pm s.d. and (range) or only range). * Calculated from data in original publication; ** number of annuli from anterior end to nerve ring, excretory pore and deirid, respectively; $\alpha$ estimated from drawings in original publication; - indicates that data is not available or not applicable; figures within [ ] indicate the number of specimens measured.

\begin{tabular}{|c|c|c|c|c|c|c|}
\hline \multirow{2}{*}{$\begin{array}{l}\text { Population } \\
\text { No. \& sex }\end{array}$} & \multicolumn{2}{|c|}{ Utah } & \multicolumn{2}{|c|}{ Kelso Dunes, California } & \multicolumn{2}{|c|}{ Italy } \\
\hline & 1 우 & $1 \hat{\jmath}$ & $\mathbf{1 0}$ 우 & 11 ठิ & 5 우 & $5 \hat{\partial}{ }^{\hat{A}}$ \\
\hline Body length & 500 & 460 & $\begin{array}{c}490 \pm 23 \\
(465-534)\end{array}$ & $\begin{array}{c}463 \pm 28 \\
(428-526)\end{array}$ & $\begin{array}{c}550 \pm 30 \\
(510-580)\end{array}$ & $\begin{array}{c}550 \pm 30 \\
(510-600)\end{array}$ \\
\hline Body diameter (BD) & $24^{*}$ & $20^{*}$ & $\begin{array}{c}23.2 \pm 1.0 \\
(22-25)\end{array}$ & $\begin{array}{c}21.2 \pm 1.7 \\
(18-24)\end{array}$ & $\begin{array}{c}29 \pm 1.4 \\
(27.5-30.8)\end{array}$ & $\begin{array}{c}25.3 \pm 2.6 \\
(20.9-27.5)\end{array}$ \\
\hline Pharynx length & $139 *$ & $115^{*}$ & $\begin{array}{c}130.6 \pm 1.0 \\
(115-141)\end{array}$ & $\begin{array}{l}123.9 \pm 7.9 \\
(107-135)\end{array}$ & $\begin{array}{l}181.4 \pm 6.1 \\
(177-192)\end{array}$ & $\begin{array}{c}184.8 \pm 24.2 \\
(161-225)\end{array}$ \\
\hline Tail length & $34 *$ & $34^{*}$ & $\begin{array}{c}38.8 \pm 1.7 \\
(32-41)\end{array}$ & $\begin{array}{c}32.2 \pm 2.5 \\
(29-36)\end{array}$ & $\begin{array}{l}50.1 \pm 3.6 \\
(44-53.9)\end{array}$ & $\begin{array}{c}48.6 \pm 3.6 \\
(45.1-53.9)\end{array}$ \\
\hline Anal or cloacal diam. (ABD) & - & - & $\begin{array}{c}14.4 \pm 0.2 \\
(14.0-14.5)\end{array}$ & $\begin{array}{c}16.1 \pm 1.0 \\
(14.5-18.0)\end{array}$ & $\begin{array}{c}18.4 \pm 0.4 \\
(17.6-18.7)\end{array}$ & $\begin{array}{c}22 \pm 1.1 \\
(20.9-23.1)\end{array}$ \\
\hline Vulva or Testis & - & - & $\begin{array}{c}307 \pm 14 \\
(291-335)\end{array}$ & $\begin{array}{c}269 \pm 23 \\
(223-300)\end{array}$ & - & - \\
\hline V-A/T & - & - & $\begin{array}{l}3.8 \pm 0.2 \\
(3.4-4.1)\end{array}$ & $e^{2}$ & - & - \\
\hline $\mathbf{a}$ & 21 & 23 & $\begin{array}{c}21.2 \pm 0.9 \\
(19.6-22.5)\end{array}$ & $\begin{array}{c}21.9 \pm 1.3 \\
(19.5-24.2)\end{array}$ & $\begin{array}{c}19 \pm 2 \\
(17-21)\end{array}$ & $\begin{array}{l}22 \pm 2.2 \\
(15-25)\end{array}$ \\
\hline b & 3.6 & 4 & $\begin{array}{l}3.8 \pm 0.2 \\
(3.5-4.2)\end{array}$ & $\begin{array}{l}3.7 \pm 0.2 \\
(3.4-4.3)\end{array}$ & $\begin{array}{c}3 \pm 0.1 \\
(2.8-3.2)\end{array}$ & $\begin{array}{l}2.9 \pm 0.2 \\
(2.6-3.3)\end{array}$ \\
\hline c & 15.0 & 13.5 & $\begin{array}{c}12.7 \pm 0.6 \\
(11.8-13.4)\end{array}$ & $\begin{array}{c}14.4 \pm 1.0 \\
(12.7-15.5)\end{array}$ & $\begin{array}{c}11 \pm 0.5 \\
(10.4-11.5)\end{array}$ & $\begin{array}{c}11.3 \pm 0.6 \\
(10.9-12.2)\end{array}$ \\
\hline c' & - & - & $\begin{array}{l}2.7 \pm 0.1 \\
(2.5-2.8)\end{array}$ & $\begin{array}{l}2.0 \pm 0.2 \\
(1.8-2.3)\end{array}$ & $\begin{array}{l}2.6 \pm 0.2 \\
(2.3-2.9)\end{array}$ & $\begin{array}{l}2.1 \pm 0.1 \\
(1.9-2.3)\end{array}$ \\
\hline V or $T(\%)$ & 66 & 41 & $\begin{array}{c}62.7 \pm 1.1 \\
(61-65)\end{array}$ & $\begin{array}{c}58.1 \pm 3.4 \\
(52-64)\end{array}$ & $\begin{array}{l}62.5 \pm 0.6 \\
(61.4-63)\end{array}$ & - \\
\hline Lip region diameter & - & - & $\begin{array}{l}8.3 \pm 0.3 \\
(7.5-8.5)\end{array}$ & $\begin{array}{l}8.0 \pm 0.6 \\
(7.0-8.5)\end{array}$ & - & - \\
\hline Stoma length & - & - & $\begin{array}{c}9.8 \pm 0.9 \\
(8.5-11.0)\end{array}$ & $\begin{array}{c}9.7 \pm 0.6 \\
(9-11)\end{array}$ & $\begin{array}{c}13.2 \pm 2 \\
(11-15.4)\end{array}$ & $\begin{array}{c}13.5 \pm 0.4 \\
(13.2-14.3)\end{array}$ \\
\hline Corpus length & - & - & $\begin{array}{c}83.2 \pm 2.8 \\
(78-86)[5]\end{array}$ & $\begin{array}{c}81.2 \pm 3.9 \\
(76-87)[5]\end{array}$ & $\begin{array}{c}123 \pm 5.4 \\
(117-129)\end{array}$ & $\begin{array}{c}123.6 \pm 21.5 \\
(102-159)\end{array}$ \\
\hline Isthmus length & - & - & $\begin{array}{c}34.2 \pm 2.7 \\
(29-36)[5]\end{array}$ & $\begin{array}{c}31.8 \pm 2.4 \\
(30-36)[5]\end{array}$ & $\begin{array}{c}30.3 \pm 7.5 \\
(17.6-36.3)\end{array}$ & $\begin{array}{c}32.7 \pm 4.6 \\
(25.3-37.4)\end{array}$ \\
\hline Bulb length & - & - & $\begin{array}{c}16.6 \pm 0.7 \\
(16-18)\end{array}$ & $\begin{array}{c}15.7 \pm 0.9 \\
(14.5-17.0)[6]\end{array}$ & $\begin{array}{c}20.2 \pm 2.1 \\
(17.6-23.1)\end{array}$ & $\begin{array}{c}18.8 \pm 0.6 \\
(18.1-19.8)\end{array}$ \\
\hline Bulb diameter & - & - & $\begin{array}{c}12.2 \pm 0.7 \\
(11-13)\end{array}$ & $\begin{array}{c}11.8 \pm 0.7 \\
(11-13)[6] \\
\end{array}$ & - & - \\
\hline Corpus/isthmus ratio & 3 & - & $\begin{array}{c}2.4 \pm 0.2 \\
(2.3-2.9)[5]\end{array}$ & $\begin{array}{c}2.6 \pm 0.2 \\
(2.1-2.8)[5]\end{array}$ & - & - \\
\hline Nerve ring from ant. end & - & - & $\begin{array}{c}88.5 \pm 4.5 \\
(78-94)\end{array}$ & $\begin{array}{c}90.2 \pm 7.1 \\
(78-102)\end{array}$ & $\begin{array}{c}137.5 \pm 6.3 \\
(129-144)\end{array}$ & $\begin{array}{c}141.8 \pm 23.1 \\
(118-179)\end{array}$ \\
\hline
\end{tabular}




\begin{tabular}{|c|c|c|c|c|c|c|}
\hline \multirow{2}{*}{\begin{tabular}{|l} 
Population \\
No. \& sex
\end{tabular}} & \multicolumn{2}{|c|}{ Utah } & \multicolumn{2}{|c|}{ Kelso Dunes, California } & \multicolumn{2}{|c|}{ Italy } \\
\hline & 1 우 & $1 \delta^{\lambda}$ & $\mathbf{1 0}+q$ & $11 ふ ふ$ & 5 우우 & $5 \widehat{\diamond}$ \\
\hline Excretory pore from ant. end & - & - & $\begin{array}{c}93.8 \pm 2.9 \\
(88-99)\end{array}$ & $\begin{array}{c}95.6 \pm 7.0 \\
(84-108)\end{array}$ & $122-147$ & - \\
\hline Deirid from ant. end & - & - & $\begin{array}{c}101.1 \pm 3.4 \\
(94-107)\end{array}$ & $\begin{array}{c}101.5 \pm 8.3 \\
(82-114)\end{array}$ & - & - \\
\hline $\mathbf{R}_{\mathrm{NR}}{ }^{* *}$ & - & - & $\begin{array}{l}32 \pm 1.5 \\
(30-35)\end{array}$ & $\begin{array}{c}32 \pm 2 \\
(30-36)\end{array}$ & - & - \\
\hline $\mathbf{R}_{\mathrm{EP}} * *$ & - & - & $\begin{array}{c}34 \pm 2 \\
(32-38)\end{array}$ & $\begin{array}{c}34 \pm 2 \\
(31-38)\end{array}$ & - & - \\
\hline $\mathbf{R}_{\mathrm{DEI}}^{* *}$ & - & - & $\begin{array}{c}37 \pm 2 \\
(35-41)\end{array}$ & $\begin{array}{c}37 \pm 2 \\
(34-41)\end{array}$ & - & - \\
\hline Annuli width at midbody & - & - & $2.4-3.4$ & $2.4-3.0$ & $2.0-2.5$ & - \\
\hline Annuli width anteriorly & - & - & $2.4-2.7$ & $2.2-3.0$ & - & - \\
\hline Vagina or Testis flexure length & - & - & $\begin{array}{c}7.3 \pm 0.4 \\
(7-8)\end{array}$ & $\begin{array}{c}39.5 \pm 6.7 \\
(27-48)\end{array}$ & $7-8$ & - \\
\hline Spermatheca or Spicule length & - & - & $\begin{array}{c}30.9 \pm 6.7 \\
(24-43)\end{array}$ & $\begin{array}{c}24.0 \pm 1.3 \\
(22-27)\end{array}$ & $24-26.5$ & $\begin{array}{c}34.5 \pm 1.6 \\
(31.9-36.3)\end{array}$ \\
\hline PUS or Gubernaculum length & - & - & $\begin{array}{c}27.3 \pm 2.3 \\
(24-31)\end{array}$ & $\begin{array}{c}13.2 \pm 1.1 \\
(12-16)\end{array}$ & $\begin{array}{c}30.6 \pm 5.9 \\
(22.5-36.3)\end{array}$ & $\begin{array}{c}20.4 \pm 1.4 \\
(19.8-23.1)\end{array}$ \\
\hline PUS/VBD & - & - & $\begin{array}{l}1.3 \pm 0.1 \\
(1.1-1.4)\end{array}$ & - & $0.7-1.3$ & - \\
\hline Rectum & - & - & $\begin{array}{c}16.0 \pm 1.6 \\
(13-18)\end{array}$ & - & $\begin{array}{c}21.6 \pm 0.3 \\
(16.5-24.7)\end{array}$ & - \\
\hline Rectum/ABD & - & - & $\begin{array}{l}1.1 \pm 0.1 \\
(0.9-1.2)\end{array}$ & - & - & - \\
\hline Phasmid & - & - & $\begin{array}{c}14.9 \pm 2.2 \\
(12-20)\end{array}$ & $\begin{array}{c}14.1 \pm 1.8 \\
(11-18)\end{array}$ & - & - \\
\hline Phasmid (\% of tail) & $30 x$ & $47 x$ & $\begin{array}{c}38.3 \pm 4.6 \\
(32-49)\end{array}$ & $\begin{array}{c}43.8 \pm 4.7 \\
(37-50)\end{array}$ & $15-30$ & $25-35$ \\
\hline
\end{tabular}

on anterior cloacal lip; two pairs (one ventrosublateral and one lateral) at midtail; three pairs (one lateral, one subventral and one dorsosublateral) closer to tail terminus. Phasmids located at about two-fifths to half of tail length.

\section{Remarks}

The population collected from sand in the Kelso Dunes area, Mojave Desert, California and described here, agrees in many respects with the type specimens of Stegelleta incisa described from Utah by Thorne (1937) and a bisexual population described from Italy by Orselli \& Vinciguerra (2002) under the name S. ophioglossa. One major difference is the presence of a cuticular flap on the anterior vulval lip directed posteriad and partly covering the vulval opening, as seen by SEM in the present specimens. This feature is not easily seen with light microscopy, which probably explains why it was not mentioned by Thorne (1937) in his original description. Orselli \& Vinciguerra (2002) also did not mention or illustrate it in their specimens, presumably for the same reason. 


\section{Stegelleta laterocornuta sp. nov. urn:1sid:zoobank.org:act:7669B17A-D71A-46C0-8BA0-EB92F28B7111}

Figs $1 \mathrm{~F}-\mathrm{J}$, 3, Table 2

Stegelleta sp. JB-75 - Nadler et al. 2006: 701, table 1.

\section{Diagnosis}

Stegelleta laterocornuta sp. nov. is characterised by a 379-512 $\mu \mathrm{m}$ long body in females and 365-476 $\mu \mathrm{m}$ in males; cuticle divided by regular longitudinal striations into 16 rows of blocks at midbody (excluding lateral field); lateral field with four incisures extending almost to tail terminus in females and to midtail papillae in males, two outer incisures extend to tail terminus in males; three pairs of asymmetrical lips, pairs of lips separated by U-shaped primary axils without guarding processes, secondary axils demarcated by a shallow incisure, each lip asymmetrically rectangular with a smooth margin, only lateral lips have slender acute tines; three labial probolae, bifurcated at half of their length, prongs bent toward one another apically, "snake-tongue"-shaped; pharyngeal corpus 2.3-3.0 times isthmus length; nerve ring and excretory pore at level of metacorpus to metacorpus-isthmus junction, deirids at level of isthmus; vulva without flap; spermatheca $17-31 \mu \mathrm{m}$ long; postuterine sac 7-24 $\mu \mathrm{m}$ long; spicules $21.5-23.5 \mu \mathrm{m}$ long.

\section{Etymology}

The species name is derived from the Latin lateris (= side) and cornu (= horn), referring to the long acute tine extending along the primary axil on the lateral lips.

\section{Material examined}

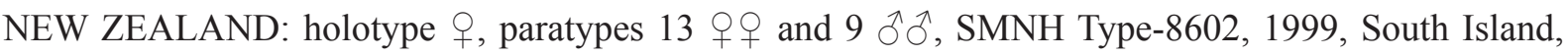
Tasman District, Abel Tasman National Park, legit Christine (Gamble) Powell, cultured as the strain Stegelleta sp. JB-75.

\section{Description}

\section{Adult}

Body slightly arcuate in different ways when killed by heat, males often strongly arcuate ventrad in tail end. Cuticle coarsely annulated, annuli $1.9-2.7 \mu \mathrm{m}$ wide at midbody and $2.0-2.4 \mu \mathrm{m}$ wide in pharyngeal region. Cuticle tessellated: longitudinal striae giving it a tiled appearance, 16 rows of blocks at midbody (excluding lateral field). Lateral field consisting of two wings separated by a broad groove, appearing as four incisures under LM, occupying about $20-25 \%$ of body diameter, extending almost to tail terminus in females and in males. Lip region slightly offset, carrying $6+4$ papillae and two round amphids. Three pairs of asymmetrical lips, one dorsal and two ventrolateral. Pairs of lips separated by U-shaped primary axils without guarding processes. Each pair with a shallow incisure demarcating a secondary axil. Each lip asymmetrically rectangular with a smooth margin. Lateral lips only with long, slender, acute tine extending along the primary axil. Three labial probolae, $7.0-8.5 \mu \mathrm{m}$ high, bifurcated at half of their length, prongs bent toward one another apically ("snake-tongue"-shaped). Stoma somewhat longer than lip region diameter. Stomatal parts not clearly discernible. Cheilorhabdia bacilliform in lateromedian view; metastegostom with a dorsal denticle. Pharynx cephaloboid. Pharyngeal corpus cylindrical; isthmus narrow, often wrinkled, and not clearly demarcated from corpus; bulb oval, with valves. Nerve ring and excretory pore at level of metacorpus-isthmus junction to isthmus. Deirids generally at level of isthmus.

\section{Female}

Reproductive system monodelphic, prodelphic, in dextral position in relation to intestine. Ovary reflexed posteriorly at oviduct, ovary straight posterior to vulva. Spermatheca well developed. Postvulval uterine 
sac about half to one-and-a-half times vulval body diameter long. Vagina about one-third of vulval body diameter. Vulval lips not or slightly protruding, without flaps. Vulva with genital plug seen in one specimen. In four females one intrauterine egg each was found, measuring 36-57 x 14.5-15.5 $\mu \mathrm{m}$. Tail conoid with 16-22 ventral annuli, terminus broadly rounded or truncate. Phasmids located at about onethird to two-fifths of tail length.

\section{Male}

Reproductive system monorchic, dextral in position; testis reflexed ventrad anteriorly. Spicules paired and symmetrical, curved ventrad; with oval manubrium and subcylindrical, gradually narrowing shaft. Gubernaculum plate-like, cornua crurum present. Tail strongly arcuate ventrad, conoid with rounded terminus. The two outer lateral lines extend posterior to the phasmid, transforming into a cuticular ridge that reaches tail terminus. Genital papillae arranged as follows: two pairs subventral precloacal (at 4-6 $\mu \mathrm{m}$ and at 23-28 $\mu \mathrm{m}$ anterior to cloaca), one pair subventral adcloacal, a single midventral papilla on anterior cloacal lip; two pairs (one ventrosublateral and one lateral) at midtail; three pairs (one lateral, one subventral and one dorsosublateral) closer to tail terminus. Phasmids located at about two-fifths to half of tail length.

\section{Remarks}

The population of Stegelleta laterocornuta sp. nov. from New Zealand described here agrees in many respects with the descriptions of S. incisa. One major difference is the presence of a long, slender and
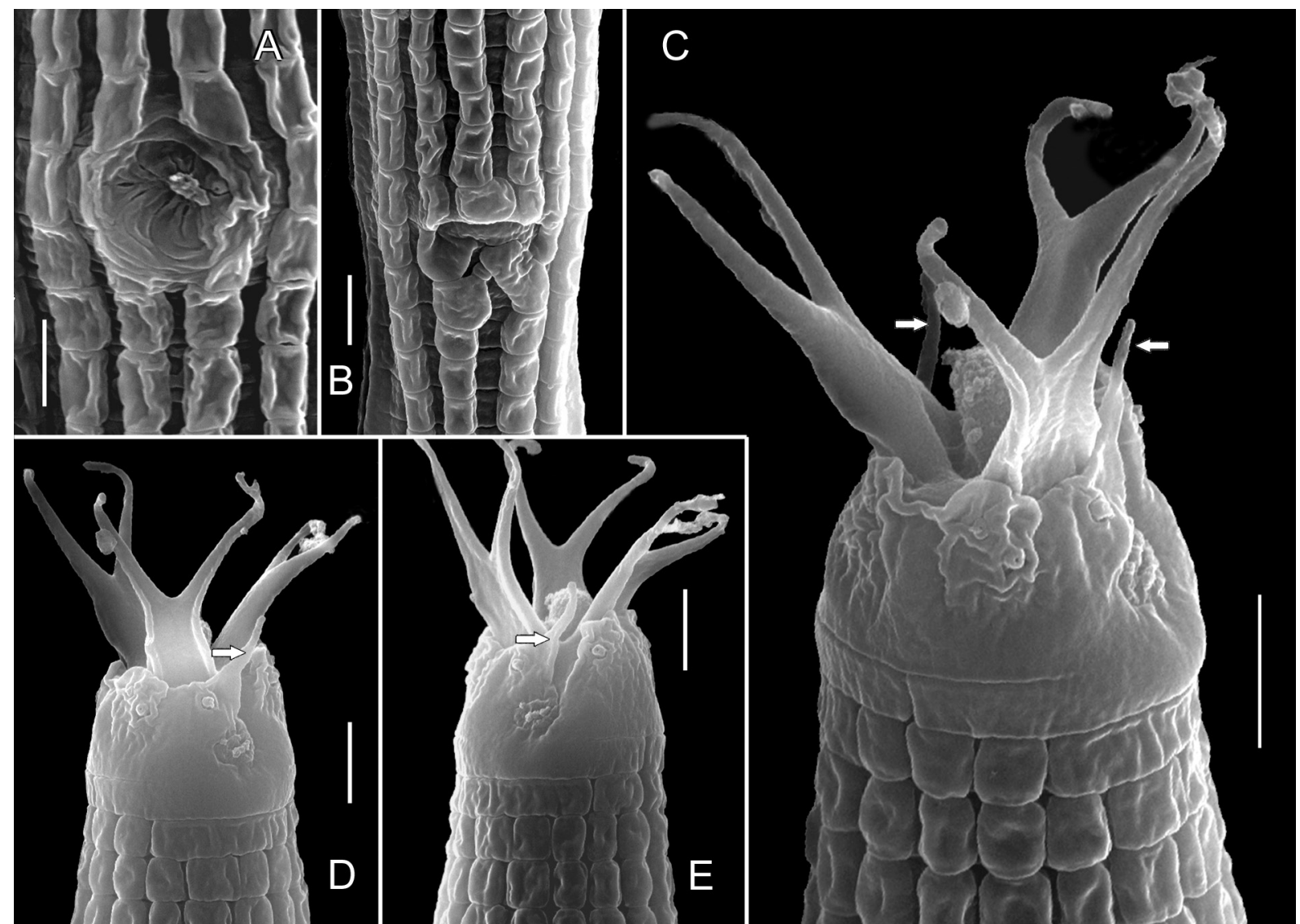

Fig. 3. Stegelleta laterocornuta sp. nov., SEM micrographs. A. Vulval opening. B. Anal opening. C-D. Anterior end, left subventral view. E. Anterior end, left lateral view (arrows in C-E point at the long acute tine extending along the primary axil on the lateral lips). Scale bars $=2 \mu \mathrm{m}$. 
Table 2. Measurements (in $\mu \mathrm{m}$ ) of Stegelleta laterocornuta sp. nov. and S. tuarua Yeates, 1967 from Castlecliff, New Zealand, the latter compared to the type population (presented as mean \pm s.d. and (range) or only range).* Number of annuli from anterior end to nerve ring, excretory pore and deirid, respectively; - indicates that data is not available or not applicable.

\begin{tabular}{|c|c|c|c|c|c|c|}
\hline \multirow{3}{*}{$\begin{array}{l}\text { Population } \\
\text { No. \& sex }\end{array}$} & \multirow{2}{*}{\multicolumn{3}{|c|}{$\begin{array}{c}\text { Stegelleta laterocornuta sp. nov. } \\
\text { New Zealand JB-75 }\end{array}$}} & \multicolumn{3}{|c|}{ Stegelleta tuarua } \\
\hline & & & & \multicolumn{2}{|c|}{ Type population } & \multirow{2}{*}{$\frac{\text { Castlecliff }}{2 \hat{\diamond}}$} \\
\hline & Holotype & $\begin{array}{c}14+q+\text { (incl. } \\
\text { holotype) }\end{array}$ & $9 \widehat{\partial} \widehat{\partial}\{1$ aberr. $\}$ & 1 우 & 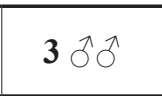 & \\
\hline Body length & 447 & $\begin{array}{c}433 \pm 41 \\
(379-512)\end{array}$ & $\begin{array}{c}428 \pm 35 \\
(365-476)\end{array}$ & 635 & $563-739$ & $650 ; 688$ \\
\hline Body diameter (BD) & 20.5 & $\begin{array}{c}19.1 \pm 1.7 \\
(15.5-21.5)\end{array}$ & $\begin{array}{c}17.4 \pm 1.1 \\
(15-19)\end{array}$ & - & - & $45 ; 46$ \\
\hline Pharynx length & 118 & $\begin{array}{l}116.7 \pm 5.2 \\
(109-126)\end{array}$ & $\begin{array}{c}117.8 \pm 4.6 \\
(109-123)\{138\}\end{array}$ & - & - & $144 ; 165$ \\
\hline Tail length & 38 & $\begin{array}{c}35.9 \pm 2.9 \\
(30-42)\end{array}$ & $\begin{array}{c}24.2 \pm 0.9 \\
(22-25)\{36\}\end{array}$ & - & - & $43 ; 48$ \\
\hline $\begin{array}{l}\text { Anal or cloacal diam. } \\
\text { (ABD) }\end{array}$ & 11 & $\begin{array}{l}11.1 \pm 0.8 \\
(9.5-12.5)\end{array}$ & $\begin{array}{c}11.9 \pm 0.4 \\
(11.0-12.5)\{13\}\end{array}$ & - & - & $30 ; 30$ \\
\hline Vulva or Testis & 273 & $\begin{array}{c}270 \pm 22 \\
(235-315)\end{array}$ & $\begin{array}{c}223 \pm 15 \\
(200-235)\end{array}$ & - & - & $432 ; 476$ \\
\hline V-A/T & 3.6 & $\begin{array}{l}3.6 \pm 0.3 \\
(3.0-3.9)\end{array}$ & - & - & - & - \\
\hline $\mathbf{a}$ & 21.8 & $\begin{array}{c}22.7 \pm 1.7 \\
(18.6-26.0)\end{array}$ & $\begin{array}{c}24.6 \pm 1.3 \\
(21.5-26.5)\end{array}$ & 21.2 & $17.2-18.8$ & $14.4 ; 15.0$ \\
\hline b & 3.8 & $\begin{array}{l}3.7 \pm 0.3 \\
(3.4-4.3)\end{array}$ & $\begin{array}{l}3.6 \pm 0.2 \\
(3.3-4.0)\end{array}$ & 3.8 & $3.9-4.8$ & $4.5 ; 4.2$ \\
\hline c & 11.8 & $\begin{array}{c}12.1 \pm 0.7 \\
(10.5-13.0)\end{array}$ & $\begin{array}{c}17.7 \pm 1.4 \\
(15.2-19.8)\{13.1\} \\
\end{array}$ & 25.4 & $14.1-16.0$ & $15.1 ; 14.3$ \\
\hline c' & 3.5 & $\begin{array}{l}3.2 \pm 0.2 \\
(2.7-3.6)\end{array}$ & $\begin{array}{c}2.0 \pm 0.1 \\
(1.8-2.2)\{2.8\}\end{array}$ & 1.6 & $1.6-1.9$ & $1.4 ; 1.6$ \\
\hline V or $\mathrm{T}(\%)$ & 61 & $\begin{array}{c}62.3 \pm 1.2 \\
(60-65)\end{array}$ & $\begin{array}{c}54.2 \pm 3.7 \\
(49-60)\end{array}$ & 69.2 & $56.8-62.7$ & $66 ; 69$ \\
\hline Lip region diameter & 7.0 & $\begin{array}{l}6.8 \pm 0.4 \\
(6.5-8.0)\end{array}$ & $\begin{array}{c}6.7 \pm 0.4 \\
(6-7)\end{array}$ & - & - & $11 ; 12$ \\
\hline Stoma length & 9.0 & $\begin{array}{l}8.9 \pm 0.3 \\
(8.5-9.5)\end{array}$ & $\begin{array}{l}8.7 \pm 0.5 \\
(8.0-9.5)\end{array}$ & - & - & $14.5 ; 19$ \\
\hline Corpus length & 70 & $\begin{array}{c}69.6 \pm 4.1 \\
(63-75)\end{array}$ & $\begin{array}{c}69.6 \pm 1.9 \\
(66-72)\{81\}\end{array}$ & - & - & $92 ; 110$ \\
\hline Isthmus length & 26 & $\begin{array}{c}26.7 \pm 2.3 \\
(24-30)\end{array}$ & $\begin{array}{c}27.1 \pm 1.7 \\
(24-29)\{36\}\end{array}$ & - & - & $22 ; 20.5$ \\
\hline Bulb length & 14.5 & $\begin{array}{c}14.8 \pm 1.0 \\
(13-17)\end{array}$ & $\begin{array}{c}14.3 \pm 0.7 \\
(13.0-15.5)\{17\}\end{array}$ & - & - & $23 ; 24$ \\
\hline Bulb diameter & 11 & $\begin{array}{l}11.5 \pm 0.7 \\
(10-13)\end{array}$ & $\begin{array}{c}11.0 \pm 0.7 \\
(10-12)\end{array}$ & - & - & $19 ; 20.5$ \\
\hline Corpus/isthmus ratio & 2.7 & $\begin{array}{l}2.6 \pm 0.2 \\
(2.3-3.0)\end{array}$ & $\begin{array}{c}2.6 \pm 0.2 \\
(2.4-2.9)\{2.3\}\end{array}$ & - & - & $4.2 ; 5.4$ \\
\hline Nerve ring from ant. end & 92 & $\begin{array}{l}83.0 \pm 7.7 \\
(70-96)\end{array}$ & $\begin{array}{c}86.6 \pm 6.4 \\
(77-96)\{102\}\end{array}$ & - & - & $108 ; 121$ \\
\hline
\end{tabular}




\begin{tabular}{|c|c|c|c|c|c|c|}
\hline \multirow{3}{*}{$\begin{array}{l}\text { Population } \\
\text { No. \& sex }\end{array}$} & \multirow{2}{*}{\multicolumn{3}{|c|}{$\frac{\text { Stegelleta laterocornuta sp. nov. }}{\text { New Zealand JB-75 }}$}} & \multicolumn{3}{|c|}{ Stegelleta tuarua } \\
\hline & & & & \multicolumn{2}{|c|}{ Type population } & \multirow{2}{*}{$\frac{\text { Castlecliff }}{2 \hat{\partial}}$} \\
\hline & Holotype & $\begin{array}{c}14+q \text { (incl. } \\
\text { holotype) }\end{array}$ & $9 \widehat{\partial} \widehat{\jmath}\{1$ aberr. $\}$ & 1 우 & $3 \widehat{\partial} \sigma^{\lambda}$ & \\
\hline $\begin{array}{l}\text { Excretory pore from ant. } \\
\text { end }\end{array}$ & 94 & $\begin{array}{c}86.3 \pm 8.1 \\
(72-97)\end{array}$ & $\begin{array}{c}92.0 \pm 6.6 \\
(81-99)\{106\}\end{array}$ & - & - & $? ; 130$ \\
\hline Deirid from ant. end & 100 & $\begin{array}{l}92.9 \pm 7.2 \\
(81-104)\end{array}$ & $\begin{array}{c}100.2 \pm 5.6 \\
(89-105)\{107\}\end{array}$ & - & - & $141 ; 171$ \\
\hline $\mathbf{R}_{\mathrm{NR}}^{*}$ & 42 & $\begin{array}{c}35 \pm 4 \\
(28-42)\end{array}$ & $\begin{array}{c}35 \pm 2 \\
(32-39)\{47\}\end{array}$ & - & - & $35 ; 38$ \\
\hline $\mathbf{R}_{\mathrm{EP}}^{*}$ & 43 & $\begin{array}{c}37 \pm 4 \\
(32-43)\end{array}$ & $\begin{array}{c}37 \pm 2 \\
(33-39)\{49\}\end{array}$ & - & - & $? ; 41$ \\
\hline $\mathbf{R}_{\mathrm{DEI}} *$ & 46 & $\begin{array}{c}40 \pm 4 \\
(35-48)\end{array}$ & $\begin{array}{c}42 \pm 2 \\
(37-44)\{50\}\end{array}$ & - & - & $46 ; 55$ \\
\hline Annuli width at midbody & 2.7 & $2.0-2.7$ & $2.0-2.7$ & - & - & $2.4 ; 2.4$ \\
\hline Annuli width anteriorly & 2.0 & $2.0-2.4$ & $2.0-2.4$ & - & - & $3.0-3.4 ; 3.0-3.4$ \\
\hline $\begin{array}{l}\text { Vagina or Testis flexure } \\
\text { length }\end{array}$ & 6.0 & $\begin{array}{l}6.0 \pm 0.6 \\
(5-7)\end{array}$ & $\begin{array}{c}39.8 \pm 3.9 \\
(35-46)\end{array}$ & - & - & 90 (dors); 82 \\
\hline $\begin{array}{l}\text { Spermatheca or Spicule } \\
\text { length }\end{array}$ & 18 & $\begin{array}{c}20.6 \pm 4.6 \\
(17-31)\end{array}$ & $\begin{array}{c}22.5 \pm 0.7 \\
(21.5-23.5)\end{array}$ & - & $36-40$ & $43 ; 42$ \\
\hline $\begin{array}{l}\text { PUS or Gubernaculum } \\
\text { length }\end{array}$ & 17 & $\begin{array}{l}18.1 \pm 5.9 \\
(7-24)\end{array}$ & $\begin{array}{c}11.4 \pm 0.5 \\
(11-12)\end{array}$ & - & $21-24$ & $25 ; 24$ \\
\hline PUS/VBD & 0.9 & $\begin{array}{l}1.0 \pm 0.3 \\
(0.6-1.3)\end{array}$ & - & - & - & - \\
\hline Rectum & 17 & $\begin{array}{c}16.6 \pm 1.1 \\
(15.5-19.0)\end{array}$ & - & - & - & - \\
\hline Rectum/ABD & 1.5 & $\begin{array}{l}1.5 \pm 0.1 \\
(1.4-1.6)\end{array}$ & - & - & - & - \\
\hline Phasmid & 12 & $\begin{array}{c}11.6 \pm 0.7 \\
(10-13)\end{array}$ & $\begin{array}{c}10.6 \pm 1.1 \\
(8.5-12.0)\{18\}\end{array}$ & - & - & $18 ; 18$ \\
\hline Phasmid (\% of tail) & 32 & $\begin{array}{c}32.2 \pm 1.7 \\
(30-37)\end{array}$ & $\begin{array}{c}44.0 \pm 3.6 \\
(38-50)\end{array}$ & - & $\sim 40$ & $42 ; 38$ \\
\hline
\end{tabular}

acute tine extending along the primary axil on the lateral lips. This is a somewhat intermediate stage between $S$. incisa, which has no tines, and $S$. arenaria, in which each lip has one acute tine extending along the primary axil. Another difference is the absence of a cuticular flap on the anterior vulval lip, as seen by SEM in the specimens of $S$. incisa from California.

\section{Stegelleta ophioglossa Andrássy, 1967}

Fig. 4A-D, Table 3

Stegelleta ophioglossa Andrássy, 1967: 208-210, fig. 3.

\section{Diagnosis}

Stegelleta ophioglossa is characterised by a 315-490 $\mu \mathrm{m}$ long body in females; cuticle divided by regular longitudinal striations into 12-16 rows of blocks at midbody (excluding lateral field); lateral field with four incisures extending almost to tail terminus in females; three pairs of asymmetrical lips, pairs of lips separated by U-shaped primary axils without guarding processes, secondary axils demarcated by 
a shallow incisure, each lip asymmetrically rectangular with a smooth margin and without tines; three labial probolae, bifurcated at half of their length, prongs bent toward one another apically, "snaketongue"-shaped; pharyngeal corpus 3-5 times isthmus length; nerve ring, excretory pore and deirids at level of isthmus; vulva without flap; spermatheca 7-13 $\mu \mathrm{m}$ long; postuterine sac 7-17 $\mu \mathrm{m}$ long.
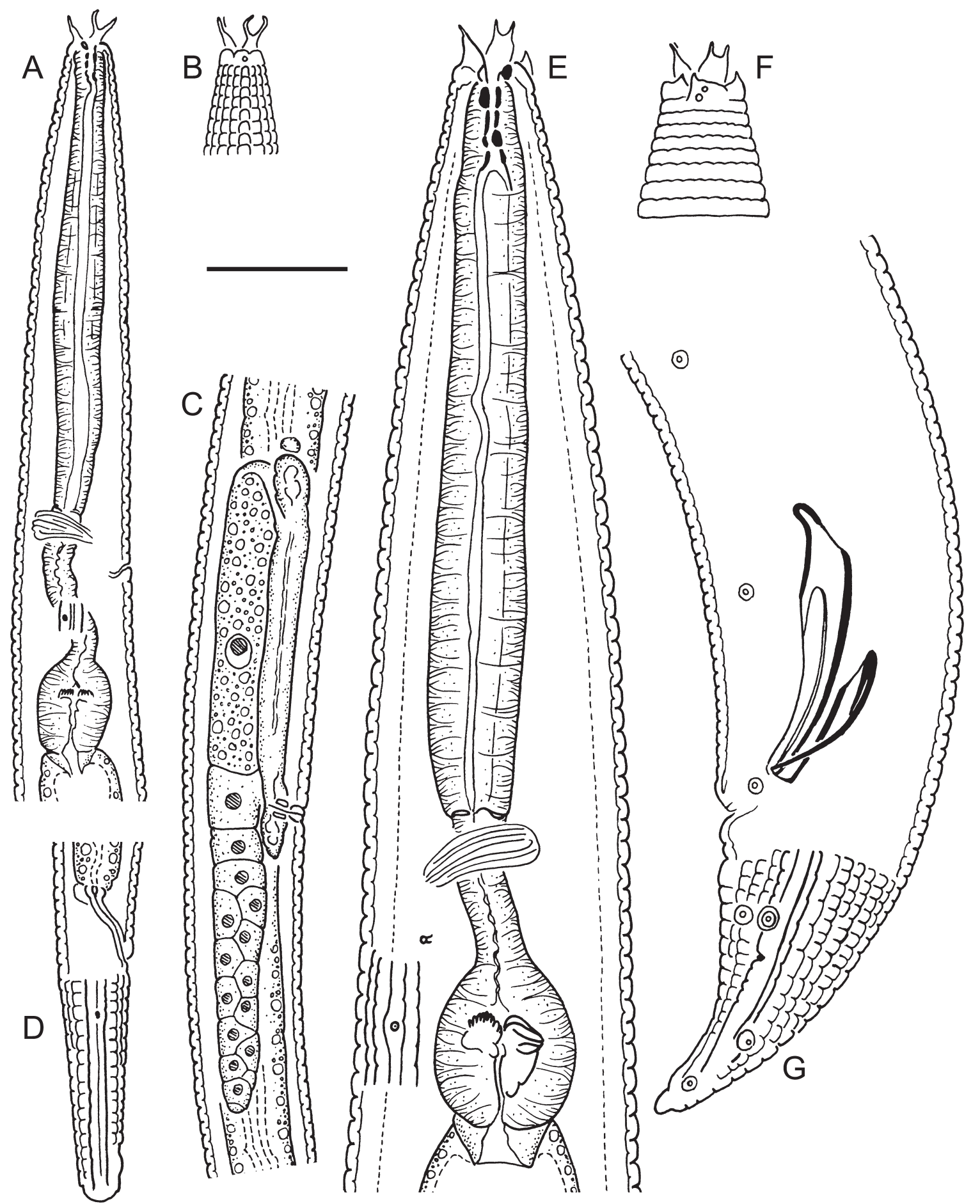

Fig. 4. A-D. Stegelleta ophioglossa Andrássy, 1967. A. Pharyngeal region. B. Anterior end, surface view. C. Female gonad. D. Female tail. E-G. Stegelleta tuarua Yeates, 1967. E. Pharyngeal region. F. Anterior end, surface view. G. Male tail. Scale bar $=20 \mu \mathrm{m}$. 
Table 3. Measurements of females of Stegelleta ophioglossa Andrássy, 1967 from Senegal compared with other populations of the same species (presented as mean \pm s.d. and (range) or only range). * Calculated from data in original publication. ** Number of annuli from anterior end to nerve ring, excretory pore and deirid, respectively; - indicates that data is not available or not applicable.

\begin{tabular}{|c|c|c|c|c|c|c|}
\hline Population & Mongolia & Uzbekistan & Senegal & Iran & Spain & Senegal \\
\hline Reference & $\begin{array}{c}\text { Andrássy } \\
1967\end{array}$ & $\begin{array}{c}\text { Mavljanov } \\
1978\end{array}$ & $\begin{array}{c}\text { De Ley et al. } \\
1990\end{array}$ & $\begin{array}{l}\text { Shokoohi } \\
\text { et al. } 2008\end{array}$ & $\begin{array}{c}\text { Abolafia } \\
\text { et al. } 2011\end{array}$ & This paper \\
\hline No. \& sex & ?우우 & 19 우 & 8 우우 & 4 우우 & $?$ 우우 & 6 우우 \\
\hline Body length & $470-490$ & $335-390$ & $\begin{array}{c}347 \pm 16 \\
(324-356)\end{array}$ & $\begin{array}{l}347 \pm 17.5 \\
(324-356)\end{array}$ & $330-420$ & $\begin{array}{c}353 \pm 22 \\
(315-382)\end{array}$ \\
\hline Body diameter (BD) & - & $17.7^{*}$ & $15-18$ & $\begin{array}{c}16.1 \pm 1.2 \\
(15-18)\end{array}$ & - & $\begin{array}{c}17.9 \pm 1.5 \\
(15.5-20.5)\end{array}$ \\
\hline Pharynx length & - & $114.7^{*}$ & $\begin{array}{c}104 \pm 5 \\
(99-111)\end{array}$ & $\begin{array}{c}93.9 \pm 3.2 \\
(90-98)\end{array}$ & - & $\begin{array}{c}102.7 \pm 3.8 \\
(97-107)\end{array}$ \\
\hline Tail length & - & $34.8^{*}$ & $\begin{array}{c}31 \pm 2 \\
(28-33)\end{array}$ & $\begin{array}{c}30.0 \pm 1.5 \\
(29-32)\end{array}$ & $27-32$ & $\begin{array}{c}31.6 \pm 1.8 \\
(29-34)\end{array}$ \\
\hline Anal body diam. (ABD) & - & 10 & $9-10$ & $\begin{array}{c}9.6 \pm 0.7 \\
(9-10)\end{array}$ & - & $\begin{array}{c}10.8 \pm 0.6 \\
(9.5-11.5)\end{array}$ \\
\hline Vulva & - & $253^{*}$ & - & $\begin{array}{c}219.7 \pm 12.6 \\
(209-238)\end{array}$ & - & $\begin{array}{c}224 \pm 13 \\
(200-243)\end{array}$ \\
\hline V-A/T & - & - & - & - & - & $\begin{array}{l}3.1 \pm 0.1 \\
(2.8-3.2)\end{array}$ \\
\hline $\mathbf{a}$ & $20-21$ & $18.1-22.9$ & $\begin{array}{c}21.3 \pm 1.2 \\
(19.7-23.1)\end{array}$ & $\begin{array}{c}21.6 \pm 0.6 \\
(20.8-21.9)\end{array}$ & $19-23$ & $\begin{array}{c}19.8 \pm 0.8 \\
(18.6-20.4)\end{array}$ \\
\hline b & $3.3-3.4$ & $3.1-3.6$ & $\begin{array}{l}3.3 \pm 0.1 \\
(3.2-3.5)\end{array}$ & $\begin{array}{l}3.7 \pm 0.1 \\
(3.7-3.8)\end{array}$ & $3.2-3.6$ & $\begin{array}{l}3.4 \pm 0.2 \\
(3.2-3.7)\end{array}$ \\
\hline c & $11-12$ & $10.4-11.8$ & $\begin{array}{c}11.2 \pm 0.6 \\
(10.4-12.9)\end{array}$ & $\begin{array}{c}11.6 \pm 0.2 \\
(11.3-11.8)\end{array}$ & $12-13$ & $\begin{array}{c}11.2 \pm 0.4 \\
(10.5-11.6)\end{array}$ \\
\hline c' & $3.0-3.5$ & & $\begin{array}{l}3.3 \pm 0.2 \\
(3.0-3.6)\end{array}$ & $\begin{array}{l}3.1 \pm 0.3 \\
(2.9-3.4)\end{array}$ & $2.3-2.7$ & $\begin{array}{l}2.9 \pm 0.2 \\
(2.6-3.2)\end{array}$ \\
\hline$V(\%)$ & $60-62$ & $63.0-66.8$ & $61-63$ & $\begin{array}{c}63.2 \pm 0.6 \\
(63-64)\end{array}$ & $62-63$ & $\begin{array}{c}63.5 \pm 0.8 \\
(63-65)\end{array}$ \\
\hline Lip region diameter & - & - & - & $\begin{array}{c}4.9 \pm 0.7 \\
(4-6)\end{array}$ & - & $\begin{array}{c}6.6 \pm 0.3 \\
(6-7)\end{array}$ \\
\hline Stoma length & - & - & $7-9$ & $\begin{array}{c}7.8 \pm 1.2 \\
(6-8)\end{array}$ & - & $\begin{array}{l}8.8 \pm 0.3 \\
(8.5-9.0)\end{array}$ \\
\hline Corpus length & - & - & $\begin{array}{c}67 \pm 3 \\
(63-71)\end{array}$ & $\begin{array}{c}63.5 \pm 3.6 \\
(60-68)\end{array}$ & - & $\begin{array}{c}65.9 \pm 2.9 \\
(61.5-69.0)\end{array}$ \\
\hline Isthmus length & - & - & $\begin{array}{c}22 \pm 2 \\
(18-25)\end{array}$ & $\begin{array}{c}14.7 \pm 2.8 \\
(12-18)\end{array}$ & - & $\begin{array}{c}15.9 \pm 1.1 \\
(14.5-17.0)\end{array}$ \\
\hline Bulb length & - & - & $13-14$ & $\begin{array}{c}16.0 \pm 0.9 \\
(16-17)\end{array}$ & - & $\begin{array}{c}14.1 \pm 0.9 \\
(12.0-14.5)\end{array}$ \\
\hline Bulb diameter & - & - & - & - & - & $\begin{array}{l}11.3 \pm 0.9 \\
(9.5-12.0)\end{array}$ \\
\hline Corpus/isthmus ratio & - & - & 3 & $3.8-5.0$ & 3 & $\begin{array}{l}4.2 \pm 0.2 \\
(4.0-4.4)\end{array}$ \\
\hline Nerve ring from ant. end & - & - & $\begin{array}{c}68 \pm 4 \\
(60-71)\end{array}$ & $\begin{array}{c}67.7 \pm 2.9 \\
(64-71)\end{array}$ & - & $\begin{array}{l}76 \pm 3.6 \\
(69-81)\end{array}$ \\
\hline
\end{tabular}


BOSTRÖM S. \& HOLOVACHOV O., Species of Stegelleta and revision of the genus

\begin{tabular}{|c|c|c|c|c|c|c|}
\hline Population & Mongolia & Uzbekistan & Senegal & Iran & Spain & Senegal \\
\hline Excr. pore from ant. end & - & - & $\begin{array}{c}66 \pm 3 \\
(61-71)\end{array}$ & $\begin{array}{c}69.4 \pm 3.6 \\
(70-74)\end{array}$ & - & $\begin{array}{c}79.5 \pm 3.5 \\
(72-83)\end{array}$ \\
\hline Deirid from ant. end & - & - & $\begin{array}{c}75 \pm 2 \\
(72-78)\end{array}$ & $\begin{array}{c}73.6 \pm 3.3 \\
(70-78)\end{array}$ & - & $\begin{array}{c}82.3 \pm 4.0 \\
(75-87)\end{array}$ \\
\hline $\mathbf{R}_{\mathrm{NR}} * *$ & - & - & - & - & - & $\begin{array}{c}36 \pm 1 \\
(35-37)\end{array}$ \\
\hline $\mathbf{R}_{\mathrm{EP}} * *$ & - & - & - & $36-39$ & - & $\begin{array}{c}37 \pm 1 \\
(36-38)\end{array}$ \\
\hline $\mathbf{R}_{\mathrm{DEI}}^{* *}$ & - & - & - & 43 & - & $\begin{array}{c}39 \pm 1 \\
(37-40)\end{array}$ \\
\hline Annuli width at midbody & $1.7-2.0$ & 2.1 & $1.5-2.1$ & $1.7-2.3$ & - & $1.6-2.2$ \\
\hline Annuli width anteriorly & - & 1.5 & - & - & - & $1.7-2.0$ \\
\hline Vagina length & - & - & $3-5$ & $\begin{array}{c}6.2 \pm 0.6 \\
(6-7)\end{array}$ & - & $\begin{array}{l}5.8 \pm 0.3 \\
(5.5-6.0)\end{array}$ \\
\hline Spermatheca length & - & - & $\leq 13$ & $8.1(n=1)$ & 13 & $\begin{array}{c}8.9 \pm 1.7 \\
(7-12)\end{array}$ \\
\hline PUS length & - & - & $11-14$ & $\begin{array}{c}13.6 \pm 4.9 \\
(8-17)\end{array}$ & 10 & $7.0-8.5$ \\
\hline PUS/VBD & $\sim 1$ & - & $1.1-1.4$ & $0.5-0.9$ & - & $0.4-0.5$ \\
\hline Rectum & - & - & $\begin{array}{c}13 \pm 2 \\
(10-16)\end{array}$ & $\begin{array}{c}12.7 \pm 0.8 \\
(12-14)\end{array}$ & - & $\begin{array}{l}12.7 \pm 1.5 \\
(9.5-14.5)\end{array}$ \\
\hline Rectum/ABD & $1.7-2.0$ & - & - & $1.3-1.4$ & $>1$ & $\begin{array}{c}1.2 \pm 0.1 \\
(1.0-1.3)\end{array}$ \\
\hline Phasmid & - & - & - & - & - & $\begin{array}{l}8.8 \pm 0.4 \\
(8.5-9.5)\end{array}$ \\
\hline Phasmid (\% of tail) & $\sim 30$ & - & $21-29$ & $24-31$ & $33-46$ & $\begin{array}{c}27.5 \pm 2.4 \\
(25-32)\end{array}$ \\
\hline Rows of cuticle blocks & 14 & 16 & 16 & - & 12 & 16 \\
\hline
\end{tabular}

\section{Material examined}

SENEGAL: 6 우, SMNH 135943-135944, Kaolack region, Thyssé-Kaymor, legit C. Villenave.

\section{Description}

\section{Adult}

Body slightly arcuate ventrad when killed by heat. Cuticle annulated, annuli $1.6-2.2 \mu \mathrm{m}$ wide at midbody and 1.7-2.0 $\mu \mathrm{m}$ wide in pharyngeal region. Cuticle tessellated: longitudinal striae giving it a tiled appearance, 16 rows of blocks at midbody (excluding lateral field). Lateral field consisting of two wings separated by a narrow groove, appearing as four incisures under LM, occupying about $20 \%$ of body diameter, extending almost to tail terminus in females. Lip region slightly offset, carrying $6+4$ papillae and two round amphids. Three pairs of asymmetrical lips, one dorsal and two ventrolateral. Pairs of lips separated by U-shaped primary axils without guarding processes. Each pair with a shallow incisure demarcating a secondary axil. Each lip asymmetrically rectangular with a smooth margin. Three labial probolae, 6-7 $\mu \mathrm{m}$ high, bifurcated at half of their length, prongs bent toward one another apically ("snake-tongue"-shaped). Stoma somewhat longer than lip region diameter. Stomatal parts not clearly discernible. Cheilorhabdia bacilliform in lateromedian view; metastegostom with a dorsal denticle. Pharynx cephaloboid. Pharyngeal corpus cylindrical; isthmus narrow, not clearly demarcated from corpus; bulb oval, with valves. Nerve ring, excretory pore and deirids at level of isthmus. 


\section{Female}

Reproductive system monodelphic, prodelphic, in dextral position in relation to intestine. Ovary reflexed posteriorly at oviduct, ovary straight posterior to vulva. Spermatheca small, not developed. Postvulval uterine sac short, about one-half of vulval body diameter long. Vagina about one-third of vulval body diameter. Vulval lips not protruding. Tail conoid with 12-20 ventral annuli, terminus truncate. Phasmids located at about one-fourth to one-third of tail length.

\section{Male}

Not found.

\section{Remarks}

The specimens described here agree in many respects with the population of Stegelleta ophioglossa from Senegal described by De Ley et al. (1990) and several other populations of S. ophioglossa (Andrássy 1967; Mavljanov 1978; Shokoohi et al. 2008; Abolafia et al. 2011).

Stegelleta tuarua Yeates, 1967

Fig. 4E-G, Table 2

Stegelleta tuarua Yeates, 1967: 536-538, fig. 5.

\section{Diagnosis}

Stegelleta tuarua is characterised by a $635 \mu \mathrm{m}$ long body in females and 563-739 $\mu \mathrm{m}$ in males; cuticle divided by regular longitudinal striations into 40-44 rows of blocks at midbody; lateral field with five incisures extending almost to tail terminus in females and in males; three pairs of asymmetrical lips, pairs of lips separated by U-shaped primary axils without guarding processes, secondary axils demarcated by a shallow incisure, each lip asymmetrically rectangular with a smooth margin and one tine; three labial probolae, bifurcated at about 1/5 of their length; pharyngeal corpus 4.2-5.4 times isthmus length; nerve ring and excretory pore at level of isthmus and deirids at level of bulb; vulva and spermatheca undescribed, postuterine sac about 1 vulval body diameter long; spicules 21-25 $\mu \mathrm{m}$ long.

\section{Material examined}

NEW ZEALAND: 2 ふึَ, SMNH 135945-135946, 18 May 2008, North Island, Wanganui, Castlecliff beach, partly stabilized coastal sand dunes with Ammophila arenaria, $50 \mathrm{~cm}$ deep, legit G. Yeates.

\section{Description}

\section{Adult}

Body slightly arcuate ventrad when killed by heat. Cuticle annulated, annuli $2.4 \mu \mathrm{m}$ wide at midbody and 3.0-3.4 $\mu \mathrm{m}$ wide in pharyngeal region. Cuticle tessellated: longitudinal striae giving it a tiled appearance, tiles are not equal in size and are not arranged in straight rows, approximately 40-44 rows of blocks at midbody. Lateral field with five incisures occupying about $20 \%$ of body diameter, extending almost to tail terminus in females. Lip region slightly offset, carrying $6+4$ papillae and two round amphids. Three pairs of asymmetrical lips, one dorsal and two ventrolateral. Pairs of lips separated by U-shaped primary axils without guarding processes. Each pair with a shallow incisure demarcating a secondary axil. Each lip asymmetrically rectangular with a smooth margin and one acute tine extending along the primary axil. Three labial probolae, $7.0-8.5 \mu \mathrm{m}$ high, bifurcated at about $1 / 5$ of their length. Stoma somewhat longer than lip region diameter. Stomatal parts not clearly discernible. Cheilorhabdia bacilliform in lateromedian view; metastegostom with a dorsal denticle. Pharynx cephaloboid. Pharyngeal corpus cylindrical; isthmus narrow, not clearly demarcated from corpus; bulb oval, with valves. Nerve ring and excretory pore at level of isthmus and deirids at level of bulb. 
Male

Reproductive system monorchic, dextral in position; testis reflexed ventrad anteriorly. Spicules paired and symmetrical, curved ventrad; with oval manubrium and subcylindrical, gradually narrowing shaft. Gubernaculum wedge-shaped, cornua crurum present. Tail strongly arcuate ventrad, conoid with rounded terminus. The two outer lateral lines extend posterior to the subdorsal papilla close to tail terminus, transforming into a cuticular ridge that reaches tail terminus. Genital papillae arranged as follows: two pairs subventral precloacal (at $36 \mu \mathrm{m}$ and at $74 \mu \mathrm{m}$ anterior to cloaca), one pair subventral adcloacal, a single midventral papilla on anterior cloacal lip; two pairs (one ventrosublateral and one lateral) at midtail; three pairs (one lateral, one subventral and one dorsosublateral) closer to tail terminus. Phasmids located at about two-fifths to half of tail length.

\section{Female}

Not found in our study.

\section{Remarks}

The specimens described here agree well in main morphological and morphometric features with the original description by Yeates (1967) of the males of S. tuarua from New Zealand.

\section{Key to species}

1. Lateral field with five incisures; cuticle with over 40 longitudinal rows of blocks; labial probolae biacute apically S. tuarua Yeates, 1967

- Lateral field with three-four incisures; cuticle with 12-26 longitudinal rows of blocks; labial probolae bifurcate half of length

2. Labial probolae with minute secondary bifurcations at tips

S. iketaia Yeates, 1967

- Labial probolae without minute secondary bifurcations at tips

3. Cephalic probolae in shape of acute tines present on all six lips along the primary axils; cuticle with 24-26 longitudinal rows of blocks

S. arenaria Boström \& Holovachov, 2012

- Cephalic probolae absent, or present only on lateral lips along the primary axils; cuticle with 12-22 longitudinal rows of blocks

4. Cuticle with 22 longitudinal rows of blocks

S. georgica Bagaturija, 1973

- Cuticle with 12-16 longitudinal rows of blocks

5. Parthenogenetic species; spermatheca $7-13 \mu \mathrm{m}$ long; postuterine sac about 7-17 $\mu \mathrm{m}$ long

- Amphimictic species; spermatheca 24-43 $\mu \mathrm{m}$ long; postuterine sac about 22-36 $\mu \mathrm{m}$ long

6. Cephalic probolae absent

S. incisa (Thorne, 1937)

- Cephalic probolae setose, present on lateral lips only

S. laterocornuta sp. nov.

\section{Discussion}

Representatives of the family Cephalobidae Filipjev, 1934 are mostly terrestrial and bacteria-consuming nematodes with a worldwide distribution, including Antarctica. They occur in tropical and temperate regions as well as in hot and cold arid areas globally. They seem to be especially diverse and abundant in deserts and many species have been described from warm and dry habitats like the Namib Desert in southern Africa (see, e.g., Rashid \& Heyns 1990a, b; Rashid et al. 1990a, b) and the Mojave Desert in southern California (see, e.g., De Ley et al. 1999; Taylor et al. 2004; Waceke et al. 2005). Sand dunes 
appear to be another suitable habitat for cephalobids, as evidenced by several studies (see, e.g., Boström \& Holovachov 2012, 2013a, b; Bussau 1991; Orselli \& Vinciguerra 2002; Yeates 1967). Species of the genus Stegelleta are rather rare inhabitants of terrestrial habitats, although they occur on all continents except Antarctica. In the present study, a new species of Stegelleta, S. laterocornuta sp. nov., is described from New Zealand, which brings the total number of species in this genus to seven. Descriptions of new material of some already known species from dry areas in California and Senegal are also included. This leads to an addition of morphological data which broadens the diagnosis of Stegelleta and increases the number of character combinations useful for species identification in the genus.

\section{Acknowledgements}

The second author was supported in part by an award from the NSF Partnerships for Enhancing Expertise in Taxonomy (PEET) program grant "PEET: Training the Next Generation of Nematode Taxonomists: Applying the Tools of Modern Monography Across Free-living and Parasitic Tylenchina"(DEB-0731516). Sampling in the Mojave Desert was performed by O. Holovachov and P. De Ley under the permit \# MOJA2010-SCI-0003 from the National Park Service, United States Department of Interior. We thank D. Sturhan, G. Yeates and C. Villenave for providing us with samples and slides, and D. Bumbarger for SEM pictures of Stegelleta JB-75 ( = S. laterocornuta sp. nov.).

\section{References}

Abolafia J., Guerrero-Rodríguez P. \& Peña-Santiago R. 2011. Nematoda, Rhabditida. Fauna Ibérica 34, Museo Nacional de Ciencias Naturales, Madrid.

Andrássy I. 1967. Ergebnisse der zoologischen Forschungen von Dr. Z. Kaszab in der Mongolei. 92. Weitere Bodennematoden aus den Jahren 1964 und 1965. Opuscula Zoologica Budapestinensis 6 (2): 203-233.

Boström S. \& Holovachov O. 2012. Description of Chilodellus eremus gen. n., sp. n. and Stegelleta arenaria sp. n. (Rhabditida: Cephalobidae) from Kelso Dunes, Mojave National Preserve, California, USA. Journal of Nematode Morphology and Systematics 15: 21-31.

Boström S. \& Holovachov O. 2013a. Description of two new species of Nothacrobeles Allen \& Noffsinger, 1971 (Rhabditida: Cephalobidae) from Kelso Dunes, Mojave National Preserve, California, USA. Journal of Nematode Morphology and Systematics 16: 25-34.

Boström S. \& Holovachov O. 2013b. Description of one new species of Heterocephalobellus Rashid, Geraert \& Sharma, 1985 from Kelso Dunes, Mojave National Preserve, California, USA and Monte Desert, Usno, Argentina. Journal of Nematode Morphology and Systematics 16: 161-166.

Bussau C. 1991. Freilebende Nematoden aus Küstendünen und angrenzenden Biotopen der deutschen und dänischen Küsten. IV. Rhabditida und Tylenchida (Nematoda). Zoologischer Anzeiger 226: 114-148.

De Ley I.T., De Ley P., Baldwin J.G., Mundo-Ocampo M. \& Nadler S.A. 1999. Three new species of Nothacrobeles (Nemata: Cephalobidae) from the Mojave Desert, California. Journal of Nematology 31 (4): 482-497.

De Ley P., Geraert E. \& Coomans A. 1990. Seven cephalobids from Senegal (Nematoda: Rhabditida). Journal of African Zoology 104 (4): 287-304.

Holovachov O., De Ley I.T., Mundo-Ocampo M. \& De Ley P. 2009. Identification of Cephaloboidea (Nematoda). EUMAINE, Gent and Nematology, UC Riverside. Available from http://www.nrm.se/ download/18.9ff3752132fdaeccb6800015606/CEPHALOBOIDEA[1].pdf [accessed 19 Feb. 2014]

Mavljanov O.M. 1978. Stegelleta cylindrica sp. n. (Acrobelinae, Rhabditida), a new species of nematodes from cotton. Zoologicheskii Zhurnal 57: 1889-1891 (in Russian). 
Nadler S.A., De Ley P., Mundo-Ocampo M., Smythe A.B., Stock S.P., Adams B.J., De Ley I.T., Holovachov O. \& Baldwin J.G. 2006. Phylogeny of Cephalobina (Nematoda): Molecular evidence for recurrent evolution of probolae and incongruence with traditional classifications. Molecular Phylogenetics and Evolution 40 (3): 696-711. http://dx.doi.org/10.1016/j.ympev.2006.04.005

Orselli L. \& Vinciguerra M.T. 2002. Nematodes from Italian sand dunes. 6. Two new and three rare species of Cephalobidae (Nematoda). Nematologia Mediterranea 30: 211-220.

Rashid F. \& Heyns J. 1990a. Chiloplacus and Macrolaimellus species from South West Africa/Namibia (Nematoda: Cephalobidae). Phytophylactica 22 (2): 189-199.

Rashid F. \& Heyns J. 1990b. Description of Namibinema scaphovulva n. gen., n. sp. and Zeldia punctata (Thorne, 1925) from Namibia (Nematoda: Cephalobidae). Phytophylactica 22 (4): 397-403.

Rashid F., Heyns J. \& Coomans A.1990a. Paracrobeles and Acrobeles species from South West Africa/ Namibia with a description of a new Acrobeles species (Nematoda: Cephalobidae). Phytophylactica 22 (1): 41-49.

Rashid F., Heyns J. \& Coomans A.1990b. Species of Seleborca from South West Africa/Namibia (Nematoda: Cephalobidae). Phytophylactica 22 (1): 51-62.

Shokoohi E., Abolafia J., Kheiri A. \& Zad J. 2008. Nematodes of the order Rhabditida from Tehran province (Iran). Some known species of the family Cephalobidae. Journal of Nematode Morphology and Systematics 11 (1): 67-85.

Taylor T.M., Baldwin J.G. \& Mundo-Ocampo M. 2004. Paracrobeles mojavicus sp. n. (Nematoda: Cephalobidae) from the Mojave Desert, California. Journal of Nematode Morphology and Systematics 6 (2): 151-160.

Thorne G. 1937. A revision of the nematode family Cephalobidae Chitwood and Chitwood, 1934. Proceedings of the Helminthological Society of Washington 4 (1): 1-16.

Thorne G. 1938. Notes on free-living and plant-parasitic nematodes. IV. Proceedings of the Helminthological Society of Washington 5 (2): 64-65.

Waceke J.W., Bumbarger D.J., Mundo-Ocampo M., Subbotin S.A. \& Baldwin J.G. 2005. Zeldia spannata sp. n. (Nematoda: Cephalobidae) from the Mojave Desert, California. Journal of Nematode Morphology and Systematics 8 (1): 57-67.

Yeates G.W. 1967. Studies on nematodes from dune sands. 5. Acrobelinae. New Zealand Journal of Science 10 (2): 527-547.

Manuscript received: 19 February 2014

Manuscript accepted: 23 April 2014

Published on: 16 June 2014

Topic editor: Rudy Jocqué

Desk editor: Charlotte Thionois

Printed versions of all papers are also deposited in the libraries of the institutes that are members of the EJT consortium: Muséum National d'Histoire Naturelle, Paris, France; Botanic Garden Meise, Belgium; Royal Museum for Central Africa, Tervuren, Belgium; Natural History Museum, London, United Kingdom; Royal Belgian Institute of Natural Sciences, Brussels, Belgium; Natural History Museum of Denmark, Copenhagen, Denmark. 